\title{
Metal abundances in hot white dwarfs with signatures of a superionized wind
}

\author{
K. Werner ${ }^{1}$, T. Rauch ${ }^{1}$, and J. W. Kruk ${ }^{2}$ \\ ${ }^{1}$ Institute for Astronomy and Astrophysics, Kepler Center for Astro and Particle Physics, Eberhard Karls University, Sand 1, \\ 72076 Tübingen, Germany \\ e-mail: werner@astro.uni-tuebingen.de \\ 2 NASA Goddard Space Flight Center, Greenbelt, MD 20771, USA
}

Received 8 August 2017 / Accepted 16 October 2017

\begin{abstract}
About a dozen hot white dwarfs with effective temperatures $T_{\text {eff }}=65000-120000 \mathrm{~K}$ exhibit unusual absorption features in their optical spectra. These objects were tentatively identified as Rydberg lines of ultra-high excited metals in ionization stages $\mathrm{V}-\mathrm{X}$, indicating line formation in a dense environment with temperatures near $10^{6} \mathrm{~K}$. Since some features show blueward extensions, it was argued that they stem from a superionized wind. A unique assignment of the lines to particular elements is not possible, although they probably stem from $\mathrm{C}, \mathrm{N}, \mathrm{O}$, and Ne. To further investigate this phenomenon, we analyzed the ultraviolet spectra available from only three stars of this group; that is, two helium-rich white dwarfs, HE 0504-2408 and HS 0713+3958 with spectral type DO, and a hydrogen-rich white dwarf, HS 2115+1148 with spectral type DAO. We identified light metals $(\mathrm{C}, \mathrm{N}, \mathrm{O}, \mathrm{Si}, \mathrm{P}$, and S) with generally subsolar abundances and heavy elements from the iron group ( $\mathrm{Cr}, \mathrm{Mn}, \mathrm{Fe}, \mathrm{Co}, \mathrm{Ni}$ ) with solar or oversolar abundance. The abundance patterns are not unusual for hot WDs and can be interpreted as the result of gravitational settling and radiative levitation of elements. As to the origin of the ultra-high ionized metals lines, we discuss the possible presence of a multicomponent radiatively driven wind that is frictionally heated.
\end{abstract}

Key words. stars: abundances - stars: atmospheres - stars: evolution - stars: AGB and post-AGB - white dwarfs

\section{Introduction}

More than two decades ago, the discovery of two hot heliumrich (DO) white dwarfs (HE 0504-2408, HS 0713+3958; effective temperature $T_{\text {eff }} \approx 70000 \mathrm{~K}$ ) with ultra-high excitation (uhe) absorption lines in optical spectra was announced (Werner et al. 1995). The newly detected lines were assigned to extremely high ionization stages of the CNO elements and neon $(\mathrm{C} \mathrm{V}, \mathrm{C}$ VI/N VI, $\mathrm{N}$ VII/O VII, O VIII, Ne IX, and Ne X). The ionization energies, however, require temperatures on the order $10^{6} \mathrm{~K}$. Some lines exhibit an asymmetric profile shape suggesting their formation in a stellar wind, hence the alternative designation of these stars as "hot-wind DOs". Since then, no progress has been made to explain the origin of these line features. However, this phenomenon turned out to be not uncommon among DO white dwarfs. In total, 9 out of about 70 known hot DOs are affected (Dreizler et al. 1995; Hügelmeyer et al. 2006; Reindl et al. 2014a; Werner et al. 2014). In addition, one DAO white dwarf (HS 2115+1148, Dreizler et al. 1995) and one PG1159 star (Hügelmeyer et al. 2006) were found to exhibit uhe lines.

The determination of the basic photospheric parameters $\left(T_{\text {eff }}\right.$ and surface gravity $g$ ) of these stars is hampered by an obviously related phenomenon in the optical spectra, namely abnormally deep He II lines, in the case of the DOs and PG1159 star, and Balmer lines, in the case of the DAO. In this paper we present the first analysis of ultraviolet (UV) spectra that are available for only three uhe stars; i.e., the two DOs and the DAO mentioned above. We performed the observations with the Goddard High Resolution Spectrograph (GHRS) aboard the
Hubble Space Telescope (HST) and the Far Ultraviolet Spectroscopic Explorer (FUSE) and use additional archival data. The primary aim of this analysis is to constrain the stellar effective temperatures by several ionization balances and determine metal abundances. Further clues concerning the uhe phenomenon are expected as well.

We introduce the program stars in Sect. 2 and present the UV observations in Sect. 3. Model atmospheres and atomic data for the spectral analysis are described in Sect. 4. Spectral line fitting procedures and results are reported in Sect. 5. The paper is concluded with a summary and discussion of the results (Sect. 6).

\section{Program stars}

Our program stars are those three WDs exhibiting uhe features, for which FUSE spectra and HST spectra with sufficient resolution spectra are available. For another DO, HS 2027+0651 (Dreizler et al. 1995), only low resolution (1.2 $\AA$ and less) HST/STIS data exist, and hence, it was not included in our study. Still another uhe DO discovered by Dreizler et al. (1995), HS 0727+6003, has not been observed with HST, and hence, we do not analyze this object in detail either. However, HS 0727+6003 has an archival, unpublished FUSE spectrum, which we will shortly discuss.

\subsection{DO white dwarfs HE 0504-2408 and HS 0713+3958}

A rough estimate of $T_{\text {eff }}=70000 \mathrm{~K}$ and $\log g=7.5$ followed from optical spectra of both stars (Werner et al. 1995). HS $0713+3958$ was reassessed by Reindl et al. (2014b) using 
A\&A 609, A107 (2018)

Table 1. Observation log of our three program stars (first three objects listed) and an additional white dwarf discussed in this paper.

\begin{tabular}{|c|c|c|c|c|c|c|c|c|c|}
\hline Star & Type & Instrument & Dataset & Grating & $R$ & $\lambda / \AA ̊$ & $t_{\exp } / \mathrm{s}$ & Date & PI \\
\hline HS $2115+1148$ & DAO & $\begin{array}{c}\text { HST/GHRS } \\
\text { HST/GHRS } \\
\text { FUSE } \\
\text { FUSE }\end{array}$ & $\begin{array}{c}\text { Z3GT0104T } \\
\text { Z3GT0105T } \\
\text { C0960101000 } \\
\text { C0960102000 }\end{array}$ & $\begin{array}{l}\text { G140L } \\
\text { G140L }\end{array}$ & $\begin{array}{c}1700-2200 \\
1700-2200 \\
20000 \\
20000\end{array}$ & $\begin{array}{c}1150-1435 \\
1480-1770 \\
915-1188 \\
915-1188\end{array}$ & $\begin{array}{l}4243 \\
5440 \\
9370 \\
6441\end{array}$ & $\begin{array}{l}1996-10-23 \\
1996-10-23 \\
2002-07-02 \\
2004-05-59\end{array}$ & $\begin{array}{l}\text { Werner } \\
\text { Werner } \\
\text { Finley } \\
\text { Finley }\end{array}$ \\
\hline HE 0504-2408 & DO & $\begin{array}{c}\text { HST/GHRS } \\
\text { HST/GHRS } \\
\text { HST/GHRS } \\
\text { FUSE }\end{array}$ & $\begin{array}{l}\text { Z2WX0104T } \\
\text { Z2WX0105T } \\
\text { Z2WX0106T } \\
\text { A0010101000 }\end{array}$ & $\begin{array}{l}\text { G140L } \\
\text { G140L } \\
\text { G270M }\end{array}$ & $\begin{array}{c}1700-2200 \\
2300-2700 \\
30000 \\
20000\end{array}$ & $\begin{array}{c}1150-1435 \\
1480-1770 \\
2957-3000 \\
915-1188\end{array}$ & $\begin{array}{l}1414 \\
2611 \\
2394 \\
6620\end{array}$ & $\begin{array}{l}1995-09-28 \\
1995-09-28 \\
1995-09-28 \\
2001-12-03\end{array}$ & $\begin{array}{l}\text { Werner } \\
\text { Werner } \\
\text { Werner } \\
\text { Werner }\end{array}$ \\
\hline HS $0713+3958$ & DO & $\begin{array}{c}\text { HST/GHRS } \\
\text { HST/STIS }^{a} \\
\text { FUSE } \\
\text { FUSE } \\
\text { FUSE }\end{array}$ & $\begin{array}{c}\text { Z2WX0204T } \\
\text { O63Z01010 } \\
\text { A0010201000 } \\
\text { A0010202000 } \\
\text { S6011901000 }\end{array}$ & $\begin{array}{l}\text { G140L } \\
\text { G750L }\end{array}$ & $\begin{array}{c}1700-2200 \\
530-1040 \\
20000 \\
20000 \\
20000\end{array}$ & $\begin{array}{c}1150-1435 \\
5240-10270 \\
915-1188 \\
915-1188 \\
915-1188\end{array}$ & $\begin{array}{l}4243 \\
2405 \\
5249 \\
9812 \\
8933\end{array}$ & $\begin{array}{l}1995-09-22 \\
2001-01-19 \\
2000-03-15 \\
2000-11-11 \\
2002-02-12\end{array}$ & $\begin{array}{c}\text { Werner } \\
\text { Werner } \\
\text { Werner } \\
\text { Werner } \\
\text { Friedman }\end{array}$ \\
\hline HS $0727+6003$ & DO & FUSE & Z9031001000 & & 20000 & $915-1188$ & 20556 & 2003-02-01 & Dupuis \\
\hline
\end{tabular}

Notes. All FUSE datasets observed with LWRS aperture. Resolving power is $R$. Exposure time is $t_{\text {exp }}{ }^{(a)}$ Observation of the cool companion.

newly available optical SDSS spectra and a grid of He+C NLTE model atmospheres, and $T_{\text {eff }}=80000 \pm 10000 \mathrm{~K}$ and $\log g=$ $7.75 \pm 0.5$ was derived. The detection of He I $\lambda 5876 \AA$ served as a $T_{\text {eff }}$ constraint and also supports our present analysis. Infrared photometry revealed that HS $0713+3958$ has a cool companion with a spectral type of late $\mathrm{K}$ that is separated about $1^{\prime \prime}$ from the primary (Napiwotzki 1997).

\subsection{HS2115+1148 (DAO)}

In their discovery paper, Dreizler et al. (1995) derived $T_{\text {eff }}=$ $67000 \mathrm{~K}$ and $\log g=6.9$ from the Balmer lines, putting emphasis on the highest observed series member $(\mathrm{H} \delta)$ and noting a severe Balmer-line problem: $\mathrm{H} \alpha-\mathrm{H} \gamma$ are much deeper in the observation than in the model with the given parameters (H+He NLTE models). Similar problems with other DAO stars (Napiwotzki \& Rauch 1994) were solved by the inclusion of C, N, and O opacities (Werner 1996), however, in this particular case the problem remained. Gianninas et al. (2010) derived $T_{\text {eff }}=62230 \mathrm{~K}, \log g=7.76$ from Balmer-line fitting using NLTE models including CNO as the only metals and assuming solar abundances. The inclusion of CNO was used as a proxy for the presence of metals to mitigate the Balmer-line problem. The two analyses arrived at similar helium abundances of $0.63 \%$ and $0.43 \%$ mass fraction, which were derived from a weak He II $\lambda 4686 \AA$ line.

Compared to the two DOs, the uhe lines in this DAO are much less prominent.

\section{Ultraviolet observations}

We obtained HST/GHRS UV spectra of the three program stars (Table 1; Figs. 1-7). Without quantitative analyses, these spectra were presented previously in Werner et al. (1997a,b), Werner \& Dreizler (1999). A high-resolution segment of HE 0504-2408 at 2957-3000 ̊ (Fig. 7) was recorded along with a red optical spectrum of the cool companion of HS 0713+3958 (Fig. 8).

Far-UV observations (915-1220 ̊) of HE 0504-2408 and HS 0713+3958 were taken with Berkeley Extreme and Far-UV Spectrometer (BEFS) aboard ORFEUS-SPAS II in 1996, but the resolution $(0.5 \AA)$ was claimed to be too poor for spectral line identification (Werner et al. 1999). A reinspection of these data in the MAST archive reveals that the spectrum of HS 0713+3958 is indeed very noisy, while the HE 0504-2408 spectrum is better but still inferior to the coadded FUSE data taken later. Hence we do not use the BEFS data. The FUSE spectra of both stars were presented by Werner et al. (2003b) and the only photospheric lines identified at that time were the $\mathrm{S}$ VI and $\mathrm{P} V$ resonance doublets. Archival FUSE data of the DAO HS 2115+1148 and DO HS $0727+6003$ are used for our work. They were hitherto unpublished and not analyzed. The FUSE data were processed using the procedure described by Werner et al. (2015). All spectra were shifted such that the photospheric lines have zero radial velocity.

The FUSE observations exhibit many absorption lines of the interstellar medium (ISM). To unambiguously identify stellar lines, we employed the program OWENS (Lemoine et al. 2002; Hébrard et al. 2002; Hébrard \& Moos 2003). It has the capacity to consider several, individual ISM clouds with their own radial and turbulent velocity, temperature, chemical composition, and respective column densities. We identified and modeled lines of H I, D I, H ${ }_{2}(J=0-9)$, HD $(J=0-1)$, C I-III, C II*, N I-II, O I, Si II, P II, S III, Ar I, and Fe II.

\section{Model atoms and model atmospheres}

We used the Tübingen Model-Atmosphere Package (TMAP ${ }^{1}$ ) to compute non-LTE, plane-parallel, line-blanketed atmosphere models in radiative and hydrostatic equilibrium (Werner \& Dreizler 1999; Werner et al. 2003a, 2012). Table 3 summarizes the number of considered non-LTE levels and radiative transitions between them. All model atoms were built from the publicly available Tübingen Model Atom Database (TMAD; Rauch \& Deetjen 2003), which is comprised of data from various sources, namely Bashkin \& Stoner (1975), the databases of the National Institute of Standards and Technology $\left(\mathrm{NIST}^{2}\right)$, the Opacity Project (OP ${ }^{3}$; Seaton et al. 1994), CHIANTI $^{4}$ (Dere et al. 1997; Landi et al. 2013), and the Kentucky Atomic Line List ${ }^{5}$.

\footnotetext{
1 http://astro.uni-tuebingen.de/ TMAP

2 http://www.nist.gov/pml/data/asd.cfm

http://cdsweb.u-strasbg.fr/topbase/topbase.html

4 http://www. chiantidatabase.org

http: //www . pa. uky. edu/ peter/atomic
} 

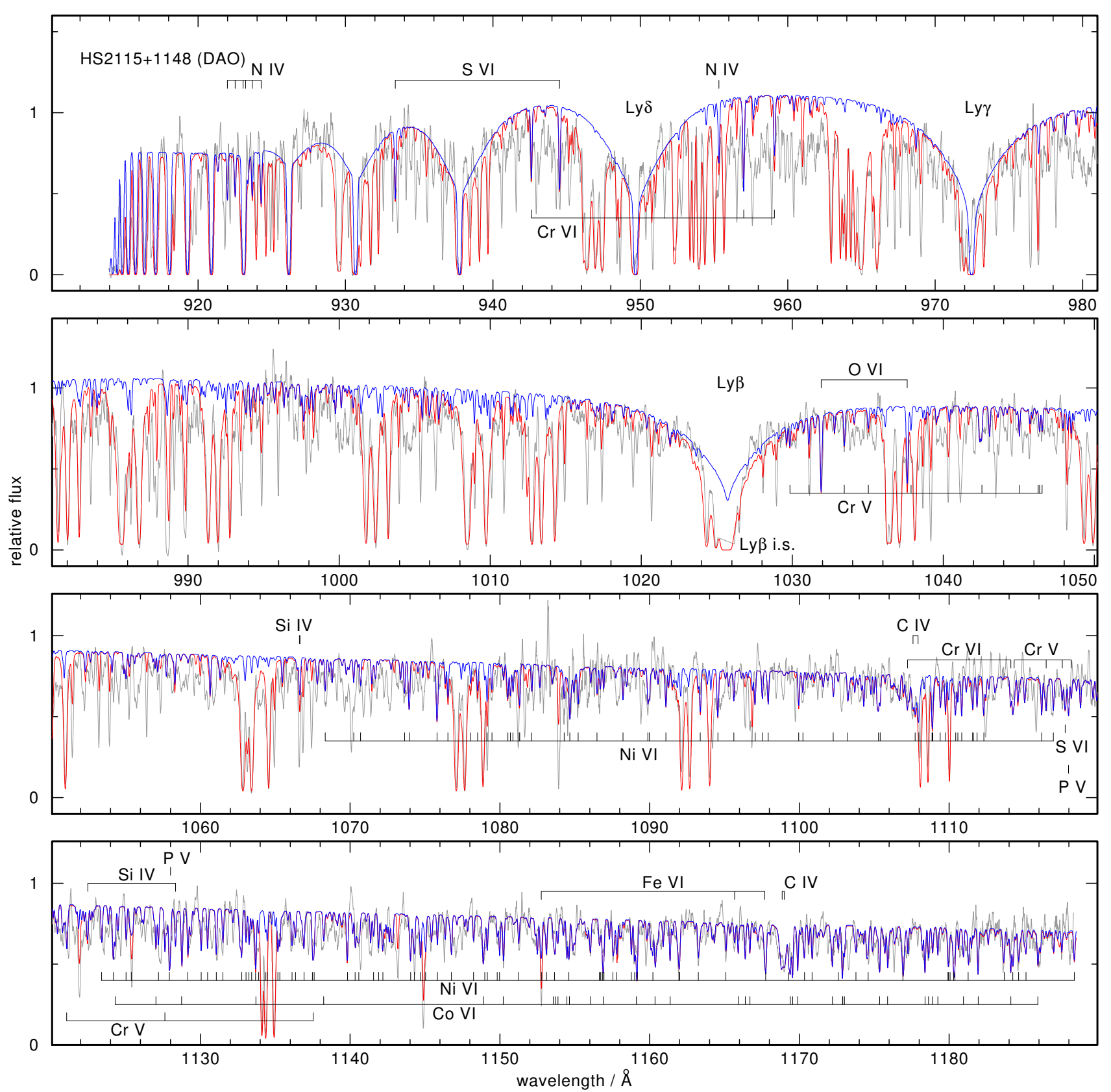

Fig. 1. FUSE spectrum of the DAO HS $2115+1148$ (gray line) compared to a photospheric model spectrum (blue line; $T_{\text {eff }}=80000 \mathrm{~K}, \log g=$ 7) with the finally adopted parameters as listed in Table 2 . The same model including interstellar absorption lines is overplotted in red. Prominent spectral lines are identified.

Besides $\mathrm{H}, \mathrm{He}, \mathrm{C}, \mathrm{N}$, and $\mathrm{O}$, our models include $\mathrm{Si}, \mathrm{P}, \mathrm{S}, \mathrm{Cr}$, $\mathrm{Mn}, \mathrm{Fe}, \mathrm{Co}$, and $\mathrm{Ni}$. To reduce the computational efforts, the light metals (up to sulfur) were considered with small model atoms and subsequently, one by one, dealt with large model atoms while keeping fixed the atmospheric structure. The statistics of these large model atoms is summarized in Table 3.

For the iron-group elements considered $(\mathrm{Cr}-\mathrm{Ni})$, we used a statistical approach, employing seven superlevels per ion linked by superlines, together with an opacity sampling method (Anderson 1989; Rauch \& Deetjen 2003). Ionization stages IV-VIII augmented by single, ground-level stages IX were considered. We used the complete line list of Kurucz (so-called LIN lists, comprising about $4.5 \times 10^{5}, 1.2 \times 10^{6}, 7.6 \times 10^{6}, 3.5 \times 10^{6}$, and $1.8 \times 10^{6}$ lines of the considered ions of $\mathrm{Cr}, \mathrm{Mn}, \mathrm{Fe}, \mathrm{Co}$, and Ni; Kurucz 1991, 2009, 2011) for the computation of the
non-LTE population numbers, and the so-called POS lists, which include only the subset of lines with well-known, experimentally observed line positions, for the final spectrum synthesis.

\section{Spectral line fitting procedure and results}

For the line profile fitting we proceeded as follows. In the 1150-1300 ̊ range of the HST spectra we assumed an arbitrary normalization factor and a reddening of $E(B-V)=0.13$ to match the continuum shape. In all other wavelength windows of the HST and FUSE spectra shown in Figs. 1-8, zero reddening was assumed and a flux scaling factor that can be different from window to window was applied. All model spectra were convolved with Gaussians according to the resolution of the observations. 


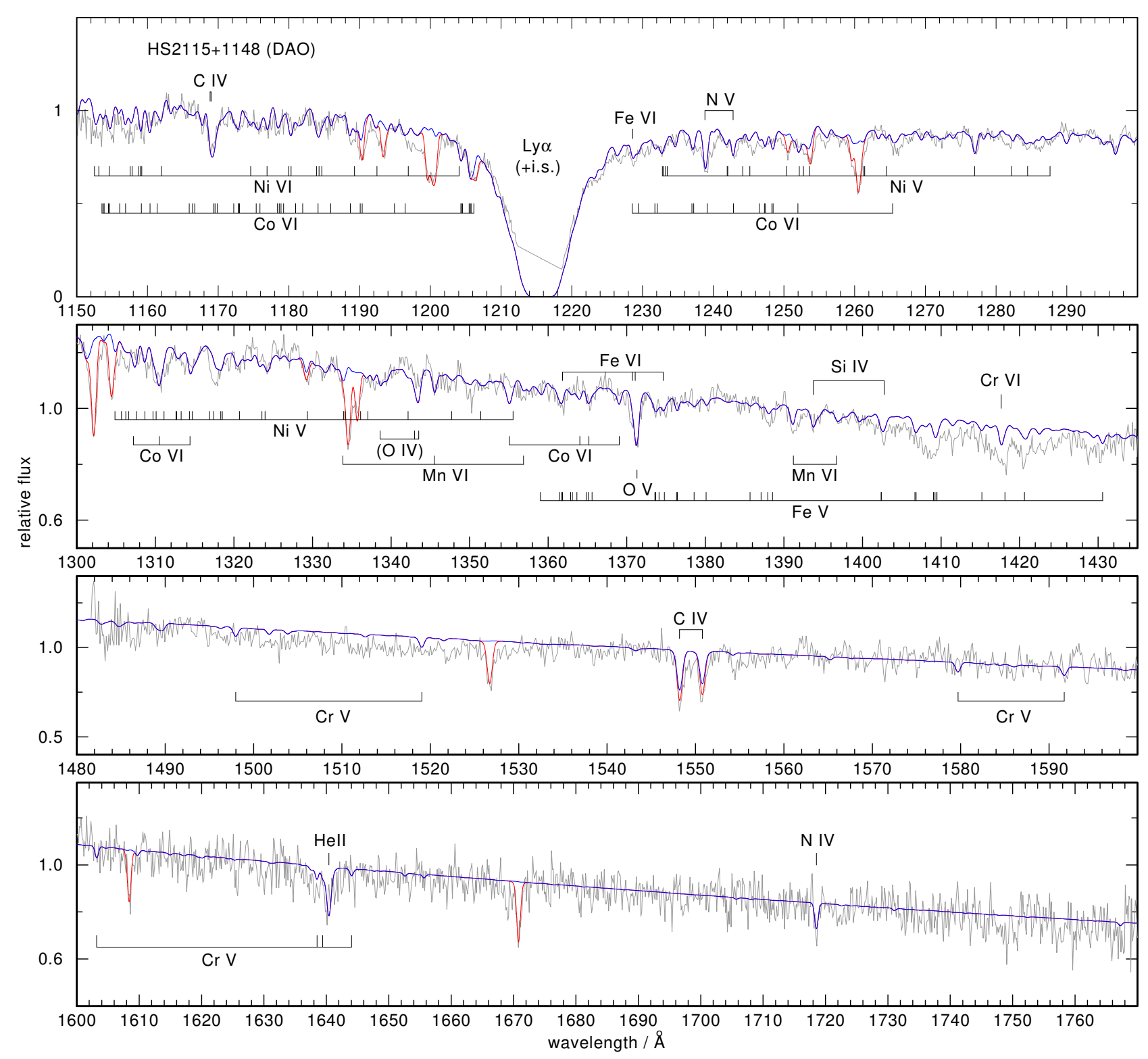

Fig. 2. HST spectrum of the DAO HS 2115+1148, similar to Fig. 1. Line identifications enclosed in brackets denote non-detections in the observations. Model: $T_{\text {eff }}=80000 \mathrm{~K}, \log g=7$.

The observed flux falls significantly below the models at about $1490-1520 \AA$ in the spectra of HE $0504-2408$ and HS $0713+3958$, which we assign to the blueshifted O VII $n=$ $5 \rightarrow 6$ uhe line (hydrogenic wavelength $1522 \AA$ ). Another broad feature at $1390-1430 \AA$ remains unexplained. In the $\mathrm{G} 270 \mathrm{M}$ spectrum of HE 0504-2408 (Fig. 7) we identify the blueshifted O VIII $n=7 \rightarrow 8$ uhe line covering the 2957-2970 ̊ interval (hydrogenic wavelength $2979 \AA$ ). This line is so broad that it is even detectable in a low-resolution (about $5 \AA$ ) IUE $^{6}$ spectrum (image LWP22367, taken from the MAST archive). The feature is also detectable in a IUE spectrum of HS 0713+3958 (LWP27020). In the same IUE spectra, the blueshifted O VII $n=6 \rightarrow 7$ uhe line (hydrogenic wavelength $2525 \AA$ ) is present in both DOs, and at shorter wavelengths the blueshifted O VIII $n=6 \rightarrow 7$ uhe line (hydrogenic wavelength $1933 \AA$ ) is just detectable (images SWP43954 and SWP49562, respectively).

\footnotetext{
6 International Ultraviolet Explorer.
}

\subsection{HS 2115+1148 (DAO)}

\subsubsection{Effective temperature and surface gravity}

At the outset, we computed a grid of hydrogen models with a small admixture of helium $(\mathrm{He}=0.004$; all element abundances in this paper are given in mass fractions) to constrain $\log g$ and $T_{\text {eff }}$ from the Lyman lines in the FUSE spectrum. The value of $T_{\text {eff }}$ was further constrained with a series of models including metals by exploiting several ionization balances (C III/IV, N IV/V, O v/VI, Fe v/VI, Ni v/VI). We finally adopted $T_{\text {eff }}=80000 \pm 5000 \mathrm{~K}$ and $\log g=7.0 \pm 0.5$. While the uncertainty in $T_{\text {eff }}$ is rather small, a tighter limit to $\log g$ is not possible. The significantly lower values for $T_{\mathrm{eff}}$ found in previous analyses of optical spectra $(67000 \mathrm{~K}$ and $62230 \mathrm{~K}$; see Sect. 2.2) are clearly excluded by our model fits to the metal lines, and the high surface gravity derived by Gianninas et al. (2010) from the Balmer lines $(\log g=7.76)$ contradicts the Lyman-line wings. In their original discovery paper, Dreizler et al. (1995) derived $\log g=6.9$ from Balmer-line fitting with pure H/He NLTE 
K. Werner et al.: Metal abundances in hot white dwarfs with signatures of a superionized wind

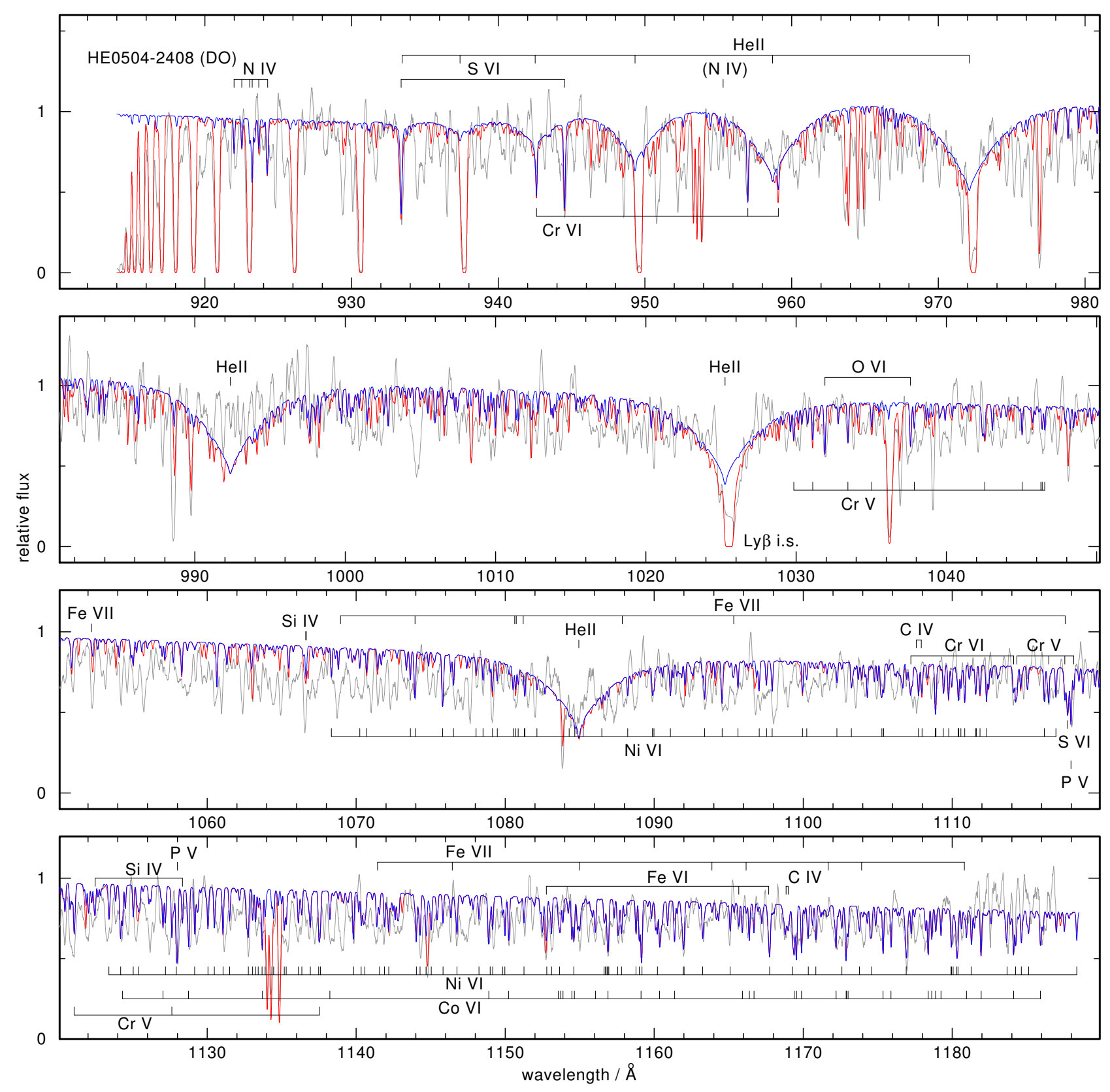

Fig. 3. FUSE spectrum of the DO HE 0504-2408, similar to Fig. 1 . Model: $T_{\text {eff }}=85000 \mathrm{~K}, \log g=7$.

models, which is close to our value; these authors note, however, the fit is poor and has a pronounced Balmer line problem.

\subsubsection{Element abundances}

Helium. He II $\lambda 1640 \AA$ suggests $\mathrm{He}=0.001$, which is lower than derived previously from He II $\lambda 4686 \AA$ (0.006 and 0.004 ; Dreizler et al. 1995; Gianninas et al. 2010). Performing a fit to He II $\lambda 4686 \AA$ in the spectrum presented by Dreizler et al. (1995) with our models confirms their high value of $\mathrm{He}=0.006$, i.e., we obtain no consistent fit to the UV and optical He II lines. At any rate, because of the low abundance, no He II lines are detectable in the FUSE spectrum.

Light metals. From a few C IV lines we find $\mathrm{C}=3.0 \times 10^{-4}$. The resonance doublet is not deep enough in the model and we suspect an interstellar contribution to the observed profile. The C III multiplet at $1175 \AA$ is not detectable, confirming the high $T_{\text {eff }}$. From a few N IV lines including $\lambda 1718 \AA$ and the $\mathrm{NV} \lambda \lambda 1239 / 1243 \AA$ resonance doublet we conclude $\mathrm{N}=3.0 \times 10^{-6}$. O V $\lambda 1371 \AA$ and the OVI $\lambda \lambda 1032 / 1038 \AA$ resonance doublet suggest $\mathrm{O}=7.0 \times 10^{-5}$. The $\mathrm{O}$ IV triplet at $1339 / 1343 / 1344 \AA$ is not detectable, again confirming the high temperature. From the Si IV $\lambda \lambda 1394 / 1403 \AA$ resonance doublet and the Si IV $\lambda \lambda 1122 / 1128 \AA$ doublet, we find $\mathrm{Si}=3.0 \times$ $10^{-5}$. From the resonance doublets P V $\lambda \lambda 1118 / 1128 \AA$ and S VI $\lambda \lambda 933 / 944 \AA, \mathrm{P}=1.0 \times 10^{-6}$ and $\mathrm{S}=5.0 \times 10^{-6}$ is derived.

Iron-group elements. The UV spectra are dominated by lines from iron and nickel. From iron we see mainly Fe V lines, which are predominantly located in the 1360-1430 $\AA$ band. In comparison, only few Fe VI lines can be identified. We derive a 


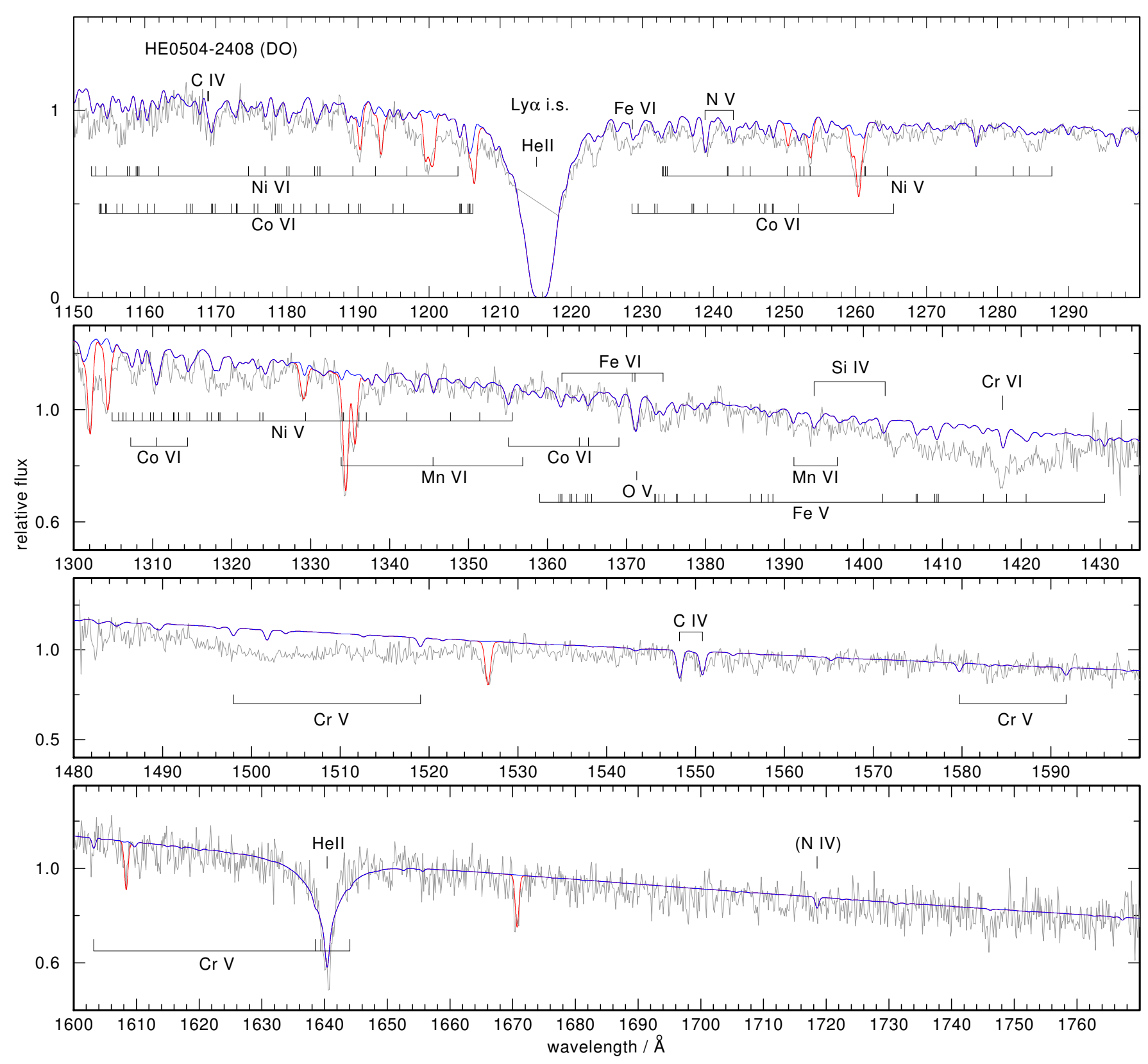

Fig. 4. HST spectrum of the DO HE 0504-2408, similar to Fig. 1 . Model: $T_{\text {eff }}=85000 \mathrm{~K}, \log g=7$.

solar abundance $\left(\mathrm{Fe}=1.3 \times 10^{-3}\right)$. From nickel, we see lines from Ni V (most of them in the 1230-1350 ̊ region) and Ni VI (1070-1200 ̊) and we adopt a 10 times solar abundance $(\mathrm{Ni}=$ $\left.7.1 \times 10^{-4}\right)$.

As indicated in Figs. 1 and 2, we identify lines from chromium, manganese, and cobalt. Specifically, we detect a smaller number of $\mathrm{CrV}$ and $\mathrm{Cr}$ VI lines and derive $\mathrm{Cr}=1.3 \times$ $10^{-3}$, which is about 2 dex oversolar. A few Mn VI lines are detectable and we measure a slightly oversolar abundance $(\mathrm{Mn}=$ $3.0 \times 10^{-5}$ ). A larger number of Co VI lines can be seen, contributing to the nickel line forest in the 1120-1260 ̊ range. The derived abundance of $\mathrm{Co}=1.0 \times 10^{-3}$ is more than two dex oversolar. The error in the abundance determinations is estimated to \pm 0.3 dex.

We comment on the many unidentified lines in this and the other program stars in Sect. 5.5 below.

\subsection{HE 0504-2408 (DO)}

\subsubsection{Effective temperature and surface gravity}

In analogy to the DAO analysis described above, we began our analysis of both DO stars with a grid of pure helium models (with a small admixture of $2.5 \times 10^{-4}$ hydrogen) to roughly constrain $T_{\text {eff }}$ and $\log g$, and then fine-tuned $T_{\text {eff }}$ by exploiting metal ionization balances. For HE 0504-2408 we found $T_{\text {eff }}=$ $85000 \pm 10000 \mathrm{~K}$ and $\log g=7.0 \pm 0.5$, i.e., similar values as for the DAO analysis, but the uncertainty in $T_{\text {eff }}$ is twice as large, mainly because of the weaker lines of $\mathrm{C}, \mathrm{N}$, and $\mathrm{O}$. A lower temperature is excluded by the lack of the C III multiplet at $1175 \AA$ and the lack of O IV lines in the HST spectrum. Fittingly, in a high-resolution $(0.1 \AA)$ optical spectrum (taken in the SPY survey; Napiwotzki et al. 2003) the He I $5876 \AA$ line is not detectable, yielding a lower limit of $T_{\text {eff }}=80000 \mathrm{~K}$. Higher 
K. Werner et al.: Metal abundances in hot white dwarfs with signatures of a superionized wind

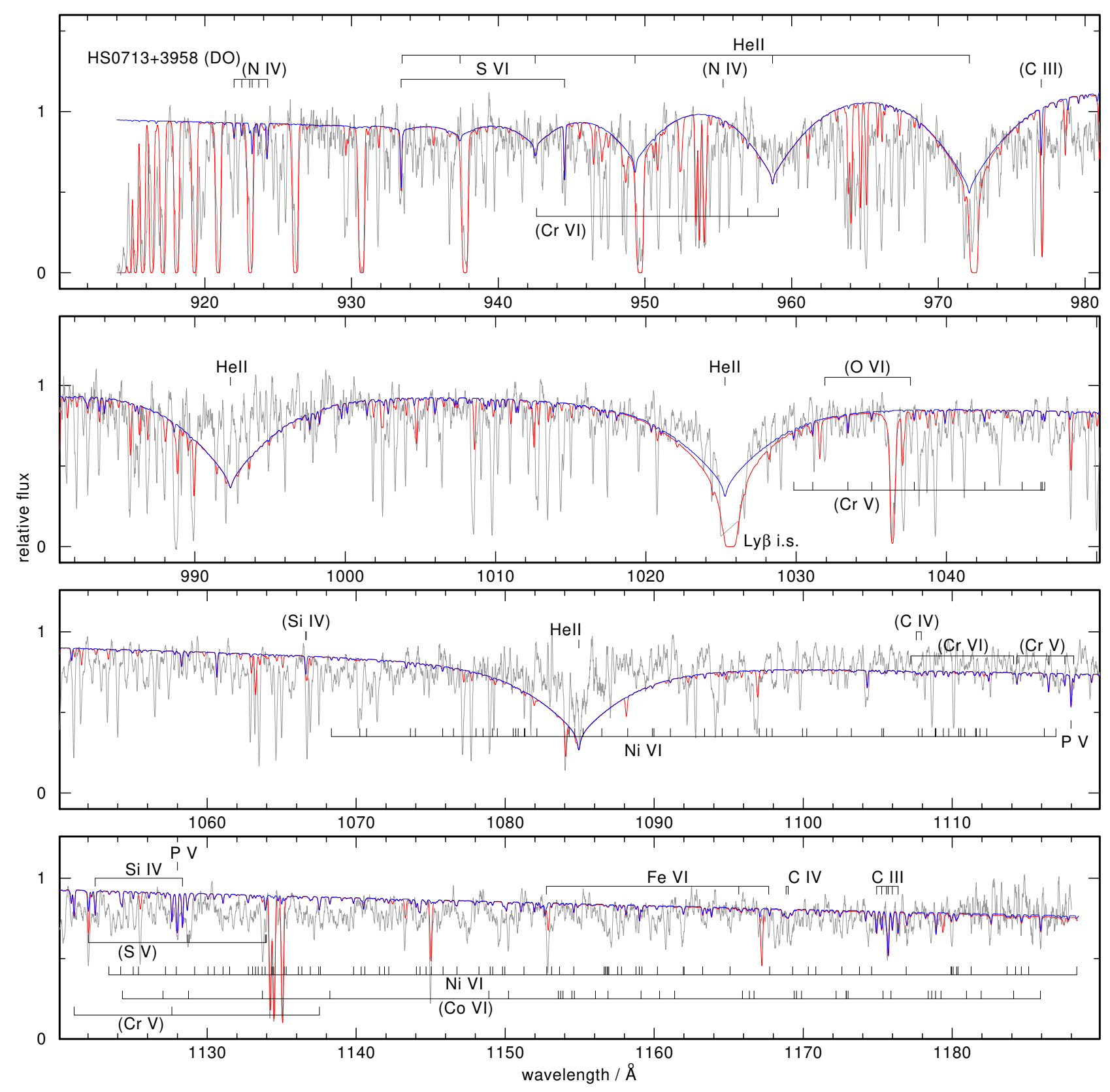

Fig. 5. FUSE spectrum of the DO HS 0713+3958, similar to Fig. 1 . Model: $T_{\text {eff }}=65000 \mathrm{~K}, \log g=7.5$.

temperatures are excluded because low-ionization heavy-metal lines would disappear ( $\mathrm{Cr} \mathrm{V}, \mathrm{Fe} \mathrm{V}, \mathrm{Ni} \mathrm{V})$. Higher surface gravities would give a too broad He II $\lambda 1640 \AA$ A line, while lower gravities would give too deep, narrow line cores in the higher series members of the Pickering series in the optical.

The model poorly fits the He II lines at $991 \AA$ and $1085 \AA$, which appear much shallower in the observation. A possible explanation is discussed in Sect. 5.5.

\subsubsection{Element abundances}

We derive $\mathrm{C}, \mathrm{N}$, and $\mathrm{O}$ abundances that are lower than in the DAO star HS $2115+1148$. For N, only an upper limit could be fixed $\left(\mathrm{C}=3.0 \times 10^{-5}, \mathrm{~N}<1.0 \times 10^{-6}, \mathrm{O}=1.0 \times 10^{-5}\right)$. While the $\mathrm{Si}$ abundance is the same $\left(3.0 \times 10^{-5}\right)$, significantly larger $\mathrm{P}$ and $\mathrm{S}$ abundances were found $\left(\mathrm{P}=1.0 \times 10^{-5}, \mathrm{~S}=5.0 \times\right.$ $10^{-5}$ ). For $\mathrm{Cr}, \mathrm{Mn}, \mathrm{Fe}, \mathrm{Co}$, and $\mathrm{Ni}$ we derive the same abundances as in the DAO analysis. As before, the error in the abundance determinations is estimated to \pm 0.3 dex.

\subsection{HS $0713+3958(D O)$}

\subsubsection{Effective temperature and surface gravity}

As mentioned in Sect. 2.1, a weak He I $5876 \AA$ line is detectable in the SDSS spectrum and serves as a good constraint for $T_{\text {eff }}$. At $\log g=7.5$ we find a best fit at $T_{\text {eff }}=65000 \pm 5000 \mathrm{~K}$. Below this temperature, He I $4471 \AA$ would be detectable but it is not visible in the observation. Reindl et al. (2014b) derived a higher temperature $\left(T_{\text {eff }}=80000 \pm 10000 \mathrm{~K}\right.$ and $\log g=$ $7.75 \pm 0.5)$ using helium-model atmospheres including only carbon. Our pure helium-models confirm this result. But in our fully metal line blanketed models, the He I $5876 \AA$ line is significantly weaker so that we arrive at a lower temperature for a good fit. 


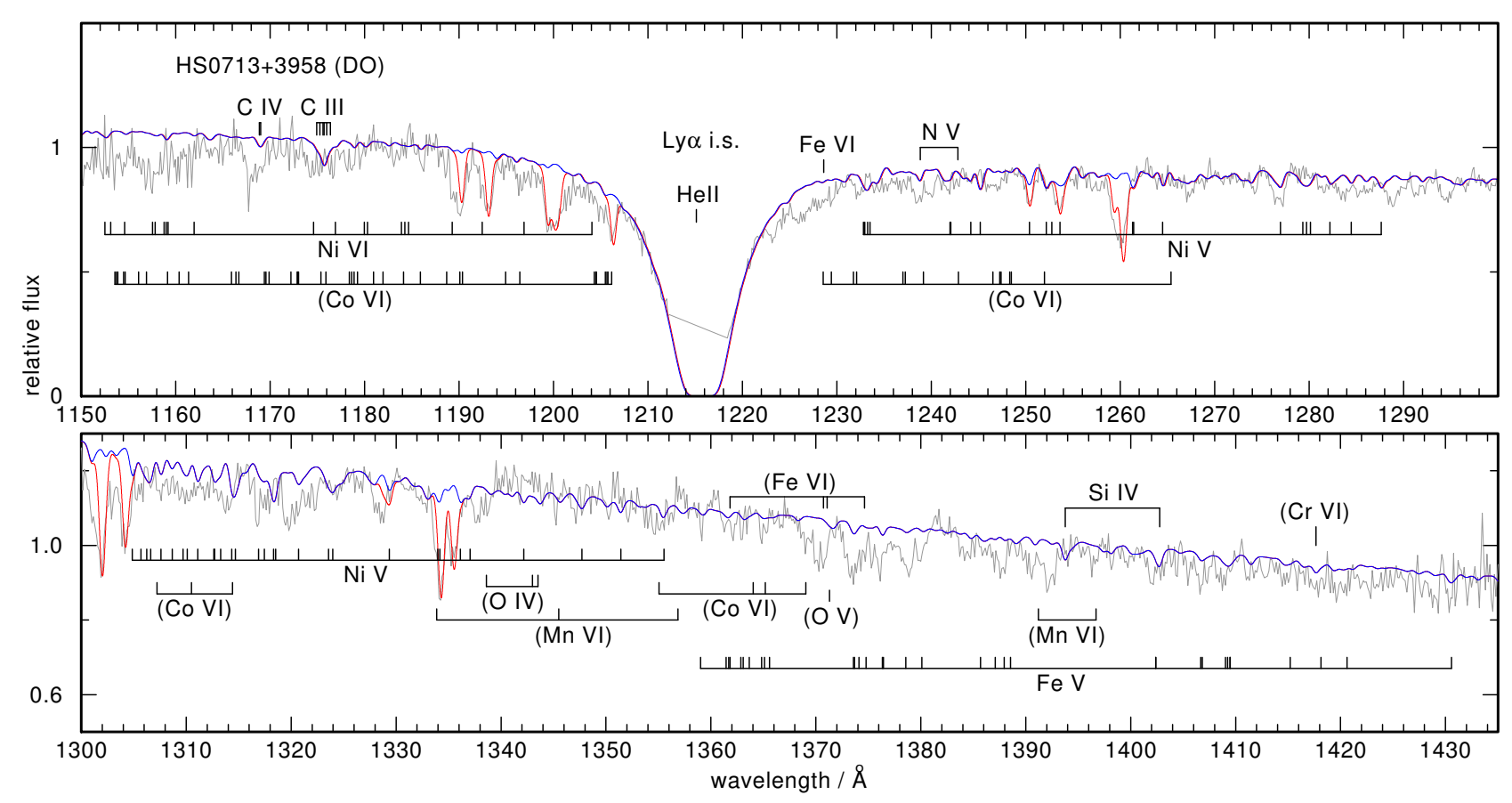

Fig. 6. HST spectrum of the DO HS 0713+3958, similar to Fig. 1 . Model: $T_{\text {eff }}=65000 \mathrm{~K}, \log g=7.5$.

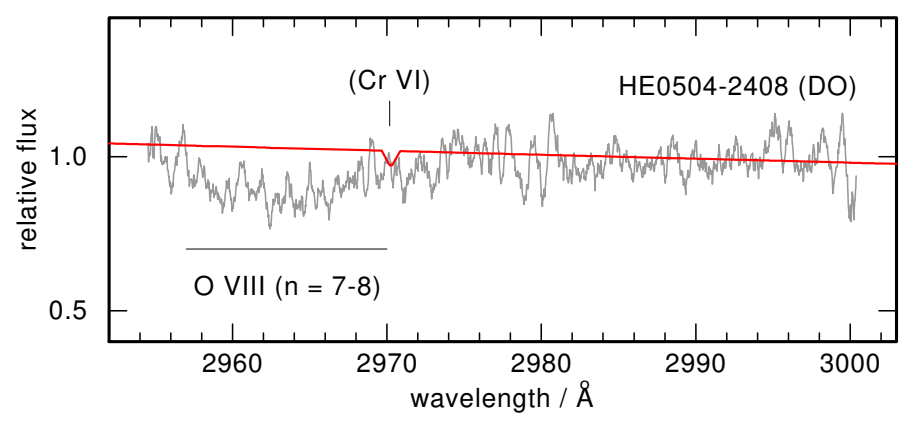

Fig. 7. HST G270M spectrum of HE 0504-2408 compared to the final model. The broad absorption feature is a uhe line as labeled.

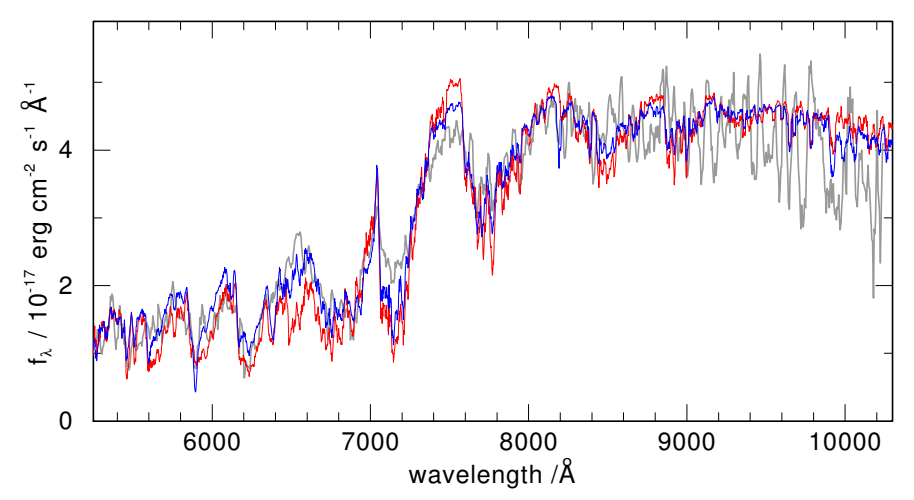

Fig. 8. HST/STIS G750L spectrum of the cool companion of HS 0713+3958 (gray line) compared to two NextGen models (blue: $T_{\text {eff }}=3300 \mathrm{~K}, \log g=5$; red: $\left.T_{\text {eff }}=3600 \mathrm{~K} ; \log g=2.5\right)$. Models and observation were smoothed with a $10 \AA$ wide boxcar.

The surface gravity cannot be fixed better from the optical spectra. For the following we assume $\log g=7.5 \pm 0.5$. The error range comprises the previously estimated values for the gravity.
Table 2. Finally adopted parameters for the three program stars.

\begin{tabular}{rrrrr}
\hline \hline & $\mathrm{HS}$ & $\mathrm{HE}$ & $\mathrm{HS}$ & \\
& $2115+1148$ & $0504-2408$ & $0713+3958$ & Sun $^{a}$ \\
\hline Type & $\mathrm{DAO}$ & $\mathrm{DO}$ & $\mathrm{DO}$ & \\
$T_{\text {eff }} / \mathrm{K}$ & 80000 & 85000 & 65000 & \\
$\log g$ & 7.0 & 7.0 & 7.5 & \\
$\mathrm{H}$ & 0.99 & - & - & 0.74 \\
$\mathrm{He}$ & $1.0 \times 10^{-3}$ & 0.995 & 0.999 & 0.25 \\
$\mathrm{C}$ & $3.0 \times 10^{-4}$ & $3.0 \times 10^{-5}$ & $7.0 \times 10^{-6}$ & $2.4 \times 10^{-3}$ \\
$\mathrm{~N}$ & $3.0 \times 10^{-6}$ & $<1.0 \times 10^{-6}$ & $<3.0 \times 10^{-7}$ & $6.9 \times 10^{-4}$ \\
$\mathrm{O}$ & $7.0 \times 10^{-5}$ & $1.0 \times 10^{-5}$ & $<3.0 \times 10^{-6}$ & $5.7 \times 10^{-3}$ \\
$\mathrm{Si}$ & $3.0 \times 10^{-5}$ & $3.0 \times 10^{-5}$ & $5.0 \times 10^{-6}$ & $6.6 \times 10^{-4}$ \\
$\mathrm{P}$ & $1.0 \times 10^{-6}$ & $1.0 \times 10^{-5}$ & $1.5 \times 10^{-7}$ & $5.8 \times 10^{-6}$ \\
$\mathrm{~S}$ & $5.0 \times 10^{-6}$ & $5.0 \times 10^{-5}$ & $1.0 \times 10^{-5}$ & $3.1 \times 10^{-4}$ \\
$\mathrm{Cr}$ & $1.3 \times 10^{-3}$ & $1.3 \times 10^{-3}$ & $<1.3 \times 10^{-4}$ & $1.7 \times 10^{-5}$ \\
$\mathrm{Mn}$ & $3.0 \times 10^{-5}$ & $3.0 \times 10^{-5}$ & - & $1.1 \times 10^{-5}$ \\
$\mathrm{Fe}$ & $1.3 \times 10^{-3}$ & $1.3 \times 10^{-3}$ & $1.3 \times 10^{-4}$ & $1.3 \times 10^{-3}$ \\
$\mathrm{Co}$ & $1.0 \times 10^{-3}$ & $1.0 \times 10^{-3}$ & $<1.0 \times 10^{-5}$ & $4.2 \times 10^{-6}$ \\
$\mathrm{Ni}$ & $7.1 \times 10^{-4}$ & $7.1 \times 10^{-4}$ & $5.0 \times 10^{-4}$ & $7.1 \times 10^{-5}$ \\
\hline & & & & \\
\hline
\end{tabular}

Notes. Abundances in mass fractions (see also Fig. 11) and surface gravity $g$ in $\mathrm{cm} \mathrm{s}^{-2}$. (a) Solar abundances from Asplund et al. (2009).

Table 3. Number of non-LTE levels and lines of model ions.

\begin{tabular}{ccccccc}
\hline \hline & I & II & III & IV & V & VI \\
\hline $\mathrm{H}$ & 15,105 & & & & & \\
$\mathrm{He}$ & 29,60 & 15,105 & & & & \\
$\mathrm{C}$ & & 1,0 & 133,745 & 54,279 & & \\
$\mathrm{~N}$ & & & 1,0 & 76,405 & 54,297 & \\
$\mathrm{O}$ & & & & 83,637 & 105,671 & 54,280 \\
$\mathrm{Si}$ & & & 17,28 & 30,102 & 25,59 & \\
$\mathrm{P}$ & & & 3,0 & 21,9 & 18,12 & \\
$\mathrm{~S}$ & & & & 39,107 & 25,48 & 38,120 \\
\hline
\end{tabular}

Notes. First and second number of each table entry denote the number of levels and lines, respectively. Not listed for each element is the highest considered ionization stage, which comprises its ground state only. See text for the treatment of iron-group elements. 
K. Werner et al.: Metal abundances in hot white dwarfs with signatures of a superionized wind
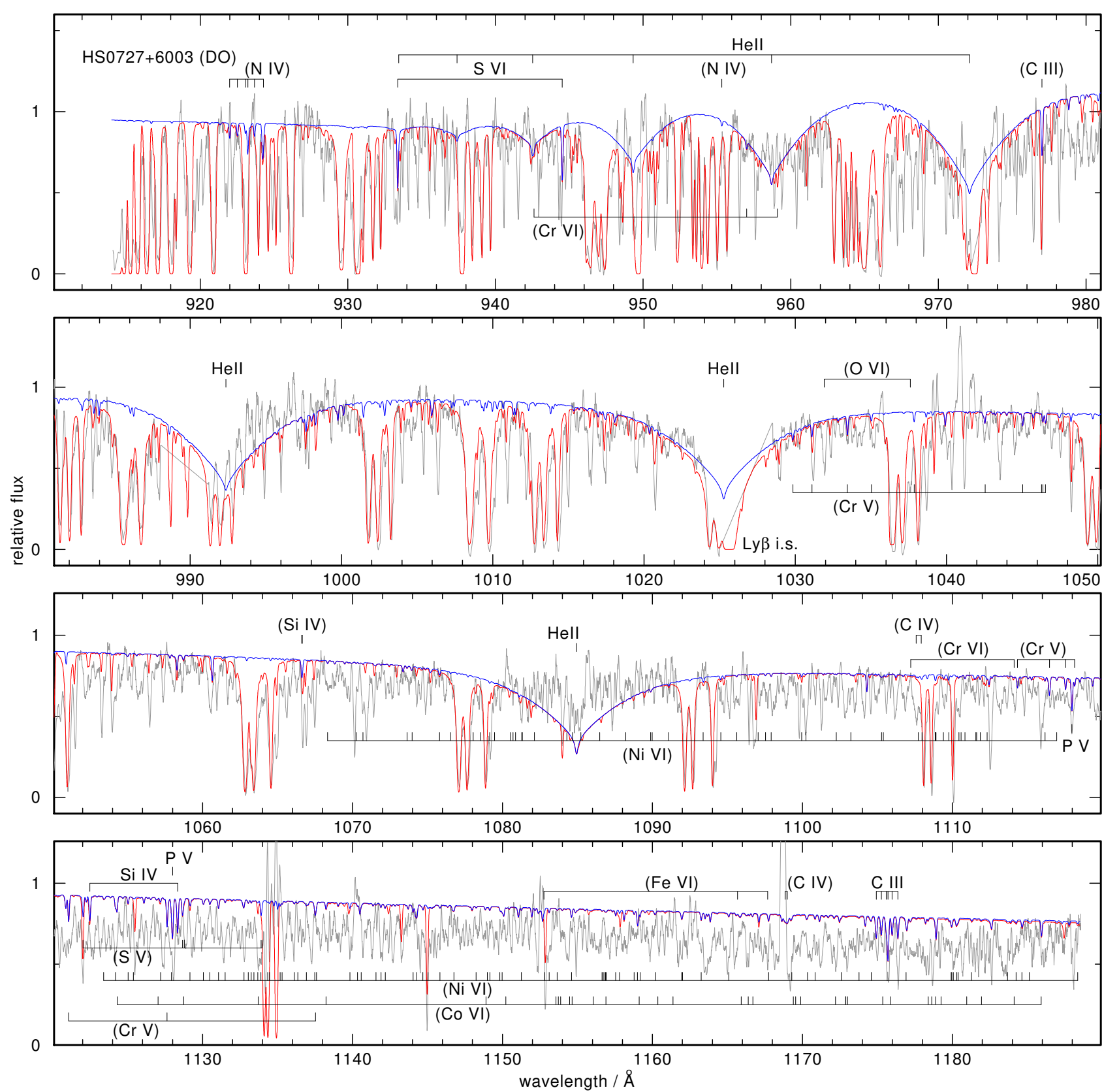

Fig. 9. FUSE spectrum of the DO HS 0727+6003, similar to Fig. 1. Model: $T_{\text {eff }}=65000 \mathrm{~K}, \log g=7.5$ (same one as for HS 0713+3958 in Fig. 5).

As was the case with the other DO, the model poorly fits the He II lines at $991 \AA$ and $1085 \AA$ because these lines are too deep to fit the observation (see discussion in Sect. 5.5).

\subsubsection{Element abundances}

Light metals. The carbon abundance is solely derived from the $\mathrm{C}$ III multiplet at $1175 \AA\left(\mathrm{C}=7.0 \times 10^{-6}\right)$. From the absence of NIV and $\mathrm{NV}$ lines and the OIV multiplet at 1338.61/1342.99/1343.51 $\mathrm{A}$ and O v $1371 \AA$, we derive $\mathrm{N}<$ $3.0 \times 10^{-7}$ and $\mathrm{O}<3.0 \times 10^{-6}$. The $\mathrm{O}$ VI resonance doublet is absent because of the low $T_{\text {eff }}$. For the other light metals we find from the same lines as discussed above $\mathrm{Si}=5.0 \times 10^{-6}$, $\mathrm{P}=1.5 \times 10^{-7}$, and $\mathrm{S}=1.0 \times 10^{-5}$. Quite large errors of 0.7 dex must be accepted because of the relatively poor quality of the spectra.
Iron-group elements. In the models, lines of $\mathrm{Cr} \mathrm{V}$ are dominant while Cr VI lines are weak. However, none of the lines can be identified in the observation and we find $\mathrm{Cr}<1.3 \times 10^{-4}$. The $\mathrm{Mn}$ VI lines seen in the two other stars are absent here because of the lower temperature. No other Mn lines are predicted so that we are unable to derive an upper abundance limit. As to iron, $\mathrm{Fe} \mathrm{V}$ lines are stronger than $\mathrm{Fe}$ VI lines in the models. We find $\mathrm{Fe}=1.3 \times 10^{-4}$. Lines from cobalt cannot be identified. Mainly from the absence of Co V lines in the HST range, we fix $\mathrm{Co}<1.0 \times 10^{-5}$. From Ni V lines we measure $\mathrm{Ni}=5.0 \times 10^{-4}$. Again, relatively large errors of 0.7 dex must be accepted.

Trans-iron elements. In the FUSE spectrum of HS 0713+3958, we possibly found lines from heavier elements but the spectrum is too noisy for firm detections. By comparison with the DO white dwarf RE 0503-289, which has similar $T_{\text {eff }}$ and $\log g(75000 \mathrm{~K}, 7.5)$ and in which 14 trans-Fe elements were 


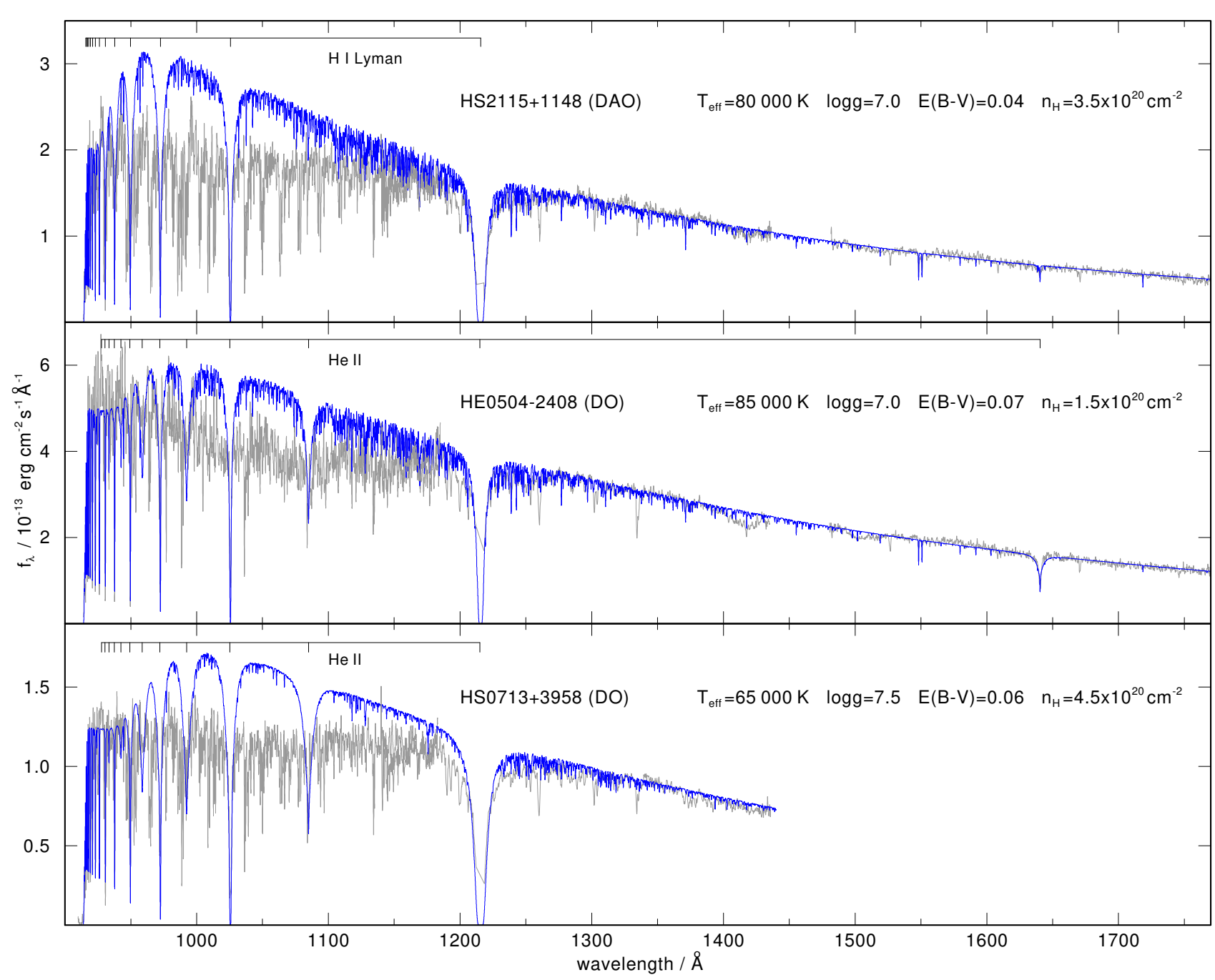

Fig. 10. Absolute flux distributions of our program stars (gray lines) compared to the final photospheric models (blue lines). The models include absorption by interstellar hydrogen Lyman lines and reddening according to the column densities $n_{\mathrm{H}}$ and $E(B-V)$ values as given in the panels. For clarity, models and observations were smoothed with Gaussians with a full width at half maximum of $0.3 \AA$.

discovered (Hoyer et al. 2017), lines from Zn v, Ge v/vI, Se vI, Mo VI, Sn V, and Te VI might be present.

\subsubsection{Spectroscopy of the cool companion}

In Fig. 8, we show the HST/STIS G750L spectrum that we took from the cool companion of HS $0713+3958$. It is characterized by TiO bands. We compare it to two NextGen (Allard et al. 2001) models (solar abundances) that suggest that $T_{\text {eff }}=3300 \mathrm{~K}$ and $\log g=5$, hence, the spectral type is M5V (Voigt 2012, p. 312; citing Aller et al. 1982), which is later than estimated previously from photometry.

The quality of the spectrum is not good enough to discern between models with different surface gravity (i.e., luminosity). A similarly good fit is achieved with a subgiant spectrum with $T_{\text {eff }}=3600 \mathrm{~K}$ and $\log g=2.5$, however, the companion must be a dwarf. The spectroscopic distance to the WD primary is $392 \mathrm{pc}$, derived from the ratio of the observed to the model flux, the surface gravity $\log g=7.5 \pm 0.5$, and the spectroscopic mass of $M_{\mathrm{WD}}=0.45 \pm 0.06 M_{\odot}$ (from extrapolation of evolutionary tracks from Althaus et al. 2009). The uncertainty in the distance is about a factor of two, which is dominated by the error in surface gravity. Taking this distance, we derive a radius of the companion of $R_{\text {comp }}=0.4 R_{\odot}$, again within a factor of two, by comparing the observed and model $\left(T_{\text {eff }}=3300 \mathrm{~K}, \log g=5\right)$ spectra. It is consistent with the radius of a M5V star $\left(0.27 R_{\odot}\right.$; Voigt 2012, p. 324).

\subsection{Archival FUSE data of HS $0727+6003$ (DO)}

As mentioned in the Introduction, we retrieved a previously unpublished FUSE spectrum of yet another uhe DO, HS $0727+6003$, from the MAST archive. It is shown in Fig. 9, together with the final model spectrum for HS $0713+3958$, which we have shown in Fig. 5. The presence of the C III multiplet at $1175 \AA$ indicates a similar temperature as that of HS $0713+3958$ $(65000 \mathrm{~K})$ but without further UV data, no detailed assessment of the photospheric parameters is possible. The only other metal lines that can be identified are from $\mathrm{Si}, \mathrm{P}$, and $\mathrm{S}$, and their strengths point at similar abundances as in HS $0713+3958$.

Two things are remarkable. First, even more discrepant than in the two analyzed DOs is the very weak He II $1085 \AA$ line, which is much deeper in the model. Second, we are unable to identify any line of iron-group elements (see discussion in Sect. 5.5). 


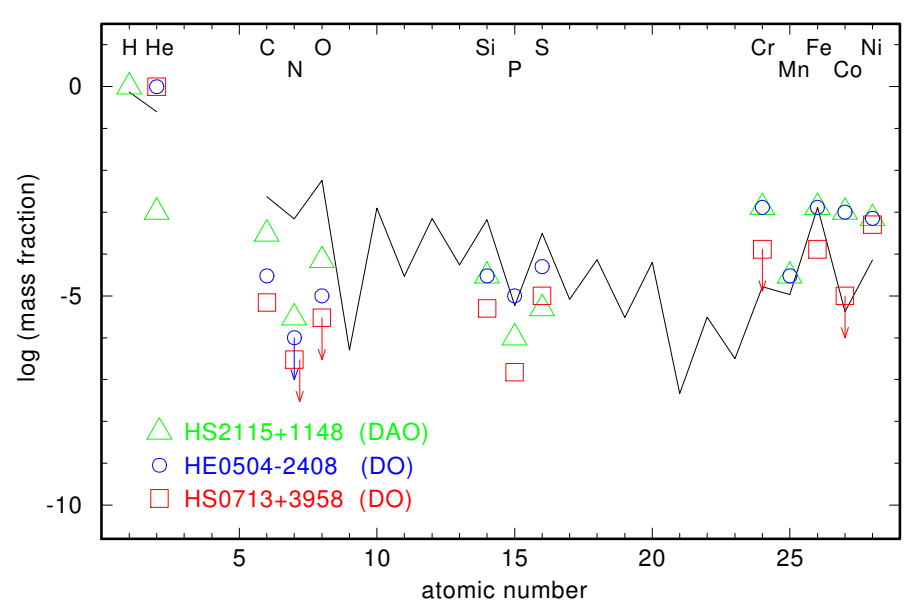

Fig. 11. Abundances measured in the three program stars. The black line indicates solar values.

\subsection{Unidentified opacities in the program stars}

In the spectra of all three program stars, particularly in the higher resolved FUSE data, many spectral lines remain unidentified. In fact, if we look at the overall flux distributions, we see that extreme blanketing by unidentified lines causes significant flux depression in the 950-1150 ̊ spectral range (Fig. 10). According to our models, they cannot be assigned to iron-group elements or other light metals. Instead, we might see either a multitude of lines from trans-iron elements (some of which are possibly identified in HS 0713+3958; see Sect. 5.3.2) and/or the flux depressions could stem from broad, unknown opacity sources, for example, from superionized species.

We ruled out that calibration problems cause the 950-1150 flux depression. The fundamental FUSE flux calibration is based on the same DA WDs as the HST spectrographs, so there is no disconnect in the definition that might lead to spurious spectral slope determinations. Furthermore, the deviation in spectral slope between the models and data begins around Ly $\alpha$ in the two hotter stars and longward of Ly $\alpha$ in the cooler HS 0713+3958, well within the STIS band. The four FUSE channels are each calibrated separately and the measured spectra in the four channels were consistent within a few percent, which is consistent with the scatter among the flux calibration measurements obtained throughout the FUSE mission. Similarly, measurements of the program stars obtained at multiple epochs were also consistent to within a few percent, indicating that there were no shortcomings in the corrections for the gradually varying throughput of the instrument. (See Sect. 7.5 of the FUSE Data Handbook for information on the FUSE flux calibration and performance throughout the mission ${ }^{7}$.)

The slope of the FUV extinction curve is known to vary from one Galactic line of sight to another, but all derived reddening laws have been monotonic shortward of about $1800 \AA$ (e.g., Sofia et al. 2005; Cartledge et al. 2005). There has been no evidence for a bump in the FUV extinction curve at $1050 \AA$ analogous to the well-known bump at $2175 \AA$.

Figure 10 also explains the weakness of the He II lines in the FUSE spectra of the DOs. In the previous figures, where we have shown the FUSE spectra, we normalized the model spectra to the local continua in the panels, however, it is obvious that the true continua are undetermined because of strong line blanketing. This finding calls into question the validity of our abundance

\footnotetext{
7 http://archive.stsci.edu/fuse/dh.html
}

determinations of metals, which exclusively rely on lines located in the range 950-1150 $\mathrm{A}$. This mainly affects phosphorus and sulfur, whose abundances could be systematically higher than we derived.

As mentioned previously (Sect. 5), there is a very shallow, roughly $20 \AA$ wide absorption feature in HE 0504-2408 centered at around $1500 \AA$. As an alternative identification as a possible uhe line, we speculated that it might be the blueshifted component of a weak C IV $\lambda 1550 \AA$ P Cygni profile (Werner et al. $2003 \mathrm{~b}$ ). If true, then it would stem from a wind with a terminal velocity of about $10000 \mathrm{~km} \mathrm{~s}^{-1}$.

\subsection{Optical spectra}

We compared our final models for the three program stars with optical spectra. In all cases, the problems with the too-deep hydrogen Balmer and He II lines remained. The problems of our models could be related to the lack of opacities from unidentified species. In the case of the DAO, the $\mathrm{H} \delta$ and $\mathrm{H} \epsilon$ lines fit well (higher series members are not present) while the lower series members are still too weak in the model, as in previous work. In the two DOs, the same problem holds for the He II Pickering lines and He II $\lambda 4686 \AA$. The relatively low $\log g=$ 7 in the models is not in contradiction to the non-detection of Pickering lines shortward of He II $\lambda 4340 \AA$. We reiterate that in the case of the DAO, we obtain inconsistent He abundances derived from the He II lines at $\lambda 1640 \AA$ and $\lambda 4686 \AA$, namely, He = 0.001 and 0.006 .

\section{Summary and discussion}

We determined metal abundances in three hot white dwarfs showing signatures of a superionized wind (see Table 2 and Fig. 11). Generally, we find low abundances of the light elements $\mathrm{C}, \mathrm{N}, \mathrm{O}, \mathrm{Si}, \mathrm{P}$, and $\mathrm{S}$ (between solar and less than $10^{-3}$ solar), while iron is solar and other Fe-group elements are between roughly solar and 100 times solar. The abundance patterns are not unusual when compared to other hot WDs with similar effective temperature. It can be assumed that they are determined by gravitational settling and radiative acceleration, although Barstow et al. (2014) argued that the wide spread of observed element abundances in hot DA white dwarfs in a given temperature range could result from accretion from external sources.

In particular, the DAO HS $2115+1148\left(T_{\text {eff }}=80000 \mathrm{~K}\right.$, $\log g=7$ ) has a metal abundance pattern that is very similar to BD-22 $3467(80000 \mathrm{~K}, 7.2$; Ziegler et al. 2012) with respect to all species. Because of the nearly identical photospheric parameters one might ask whether $\mathrm{BD}-22^{\circ} 3467$ exhibits uhe lines in the optical spectrum. Unfortunately, no optical spectrum exists because it is a close binary star with a dominating cool companion. Good et al. (2005) measured abundances of C, N, O, Si, Fe, and $\mathrm{Ni}$ in $16 \mathrm{DAO}$ white dwarfs and the results are qualitatively similar to those for HS $2115+1148$ as well.

We seek to understand how the metal abundances of the two DOs of our investigation (having $65000 \mathrm{~K}$ and $85000 \mathrm{~K}$; both with $\log g=7$ ) compare to other DOs. A recent investigation of two DOs with $\log g=7$ but significantly higher temperatures $(115000 \mathrm{~K}$ and $125000 \mathrm{~K})$ revealed solar abundances of heavier elements ( $\mathrm{Ne}, \mathrm{Si}, \mathrm{P}, \mathrm{S}, \mathrm{Ar}, \mathrm{Fe}$, and $\mathrm{Ni}$ ). This is the consequence of on-going mass loss preventing gravitational settling (Werner et al. 2017). The most detailed analyzed DO with similar temperature is RE 0503-289 $(70000 \mathrm{~K}, \log g=7.5$; 
Rauch et al. 2017). With the exception of carbon, which is oversolar in RE 0503-289, other light metals (N, O, Si, P, and S) are underabundant by about one dex, in qualitative agreement with our DOs studied here. Fe and Ni abundances in RE 0503-289 $(<0.01$ solar and solar, respectively) are lower than in our two DOs (0.1-1.0 solar and 0.9-1 solar). Aside from the enhanced $\mathrm{Fe}$ and $\mathrm{Ni}$ abundances, there is no significant difference in the abundances of the two hot-wind DOs and RE 0503-289, which has no signatures of a superhot wind.

For our analysis, we computed line-blanketed, chemically homogeneous non-LTE model atmospheres, including the opacities of all investigated metals. We hoped that these models would remove the Balmer-line problem in the DAO and the respective He II line problem in the DOs. However, the effect of the considered metal opacities on the atmospheric structure is insufficient to obtain good fits to the extraordinarily deep $\mathrm{H}$ and He II line cores in the optical spectra. This corroborates our earlier suspicion that this problem is probably related to the occurrence of the ultra-high ionization lines associated with a super-hot wind.

In the UV spectra, there is no hint as to ongoing mass loss. There is neither blueshift of nor asymmetry in the resonance line profiles (doublets of C IV, N V, O VI, Si IV, P V, S VI) or other features like OV $\lambda 1371 \AA$ and He II $\lambda 1640 \AA$ (for a possible exception concerning the CIV line, see Sect. 5.5). This is not unexpected because mass-loss rates predicted from radiationdriven wind theory are so $\operatorname{low}\left(\log \left(\dot{M} / M_{\odot} \mathrm{yr}^{-1}\right)<10^{-13}\right.$; Unglaub \& Bues 2000) that they cannot be detected spectroscopically in these lines. On the other hand, if we believe that the uhe line features are signatures of a superionized wind, then the question arises where these features are formed and how the wind is heated to temperatures on the order $10^{6} \mathrm{~K}$. The situation is reminiscent of the occurrence of "superionization" in the winds of extreme helium (EHe) stars (Hamann et al. 1982), albeit at a lower temperature scale. For example, Jeffery \& Hamann (2010) in their investigation of mass loss in the very thin wind of $\mathrm{BD}+10^{\circ} 2179\left(T_{\text {eff }}=18500 \mathrm{~K}, \log g=2.6\right)$, the observed wind profile of the CIV resonance line could not be reproduced by the models because at that low $T_{\text {eff }}$ they predicted that carbon recombines to C III and C II. It was concluded that some "superionization" keeps the ionization higher than predicted. As a viable mechanism these authors discussed frictional heating in a multicomponent wind. In fact, stellar wind models for subluminous hot stars confirm that this is well possible (Unglaub 2006; Krtička et al. 2016). In the present context of the much hotter WDs, such "superionization" is observed, albeit involving higher temperatures and, thus, higher ionization stages.

It has been predicted by models that the process of frictional heating can occur in thin radiation-driven winds of hot stars (Springmann \& Pauldrach 1992). Radiation pressure accelerates only metal ions while the bulk matter (hydrogen and/or helium) stays inert. In models for such multicomponent wind models for $\mathrm{OB}$ stars, temperatures on the order $10^{6} \mathrm{~K}$ were predicted (Krtička \& Kubát 2001). Particularly interesting for our discussion here are investigations of stellar winds with low metallicity because in the WDs of the present paper, the abundances of light metals are well subsolar. These investigations aimed at stars with low metallicity in the early Universe or even at the first stars which, in late evolutionary phases, have CNO driven winds (Krtička et al. 2010). For example, for a massive star with $T_{\text {eff }}=$ $40000 \mathrm{~K}$ and 0.001 solar metallicity, Krtička et al. (2003) find a strong increase in temperature of the photon absorbing ions to $T \approx 200000 \mathrm{~K}$ close to the stellar surface (1.14 stellar radii), while the non-absorbing bulk component (hydrogen and helium) remains at temperatures below $100000 \mathrm{~K}$. It is predicted, that the bulk material either forms clouds around the star or falls back to the stellar surface. We may speculate that the too-deep optical $\mathrm{H}$ and He II lines in our WDs are caused by relatively cool gas in such a circumstellar static cloud, while the uhe lines form in the same region but originate from the frictionally heated metal ions, which were accelerated to develop a superhot high-velocity wind. A possible scenario of pure metallic winds with hydrostatic $\mathrm{H}$ and $\mathrm{He}$ at $\log \left(\dot{M} / M_{\odot} \mathrm{yr}^{-1}\right)<10^{-16}$ has been discussed in the context of subdwarf B stars (Unglaub 2008) and this could be relevant in the superionized wind WDs as well.

Systematic studies of multicomponent wind models for hot white dwarfs are necessary to check the possibility of whether the hot-wind phenomenon is indeed caused by frictional heating in radiatively driven winds. In a first study presented by Krtička \& Kubát (2005) it can be seen, that a cooling white dwarf crosses a narrow strip in the $\log g-T_{\text {eff }}$ diagram where frictionally heated winds occur. The strip edge at low temperature is defined by the complete decoupling of the metallic wind from the bulk $\mathrm{H}+\mathrm{He}$ matter, while the strip edge at high temperature results from the transition to chemically homogeneous winds because of high mass-loss rates. This strip could explain the fact that the hot-wind WD phenomenon is restricted to WDs in the range $T_{\text {eff }}=65000-120000 \mathrm{~K}$. Such a systematic study could also answer the question why this phenomenon predominantly affects helium-dominated WDs, while only one affected (He/C/O-dominated) PG1159 star and one H-rich WD are known.

We close by mentioning that the first example of a star with a fractionated wind is the Bp star $\sigma$ Ori E (Groote \& Hunger 1997). This star is an oblique magnetic rotator whose field supports two corotating circumstellar clouds, fed by the photospheric wind, and located at the intersections of the magnetic and rotational equators. The gas temperature in the clouds is similar to the stellar effective temperature and the clouds are opaque in the lines and continuum (Smith \& Bohlender 2007), as derived from variations of high-level Balmer lines first discussed by Groote \& Hunger (1976, 1977). The gas further expands into an outer, rotational-phase independent, coronal wind and, according to Havnes \& Goertz (1984), could attain temperatures of $10^{5}-10^{7} \mathrm{~K}$. We may speculate that such a rigidly rotating magnetosphere model with an ambient, very hot coronal wind might be related to the uhe lines in the WDs discussed in the present paper.

$\sigma$ Ori $\mathrm{E}$ is variable, both spectroscopically and photometrically. The period of about 1.19 days (Hesser et al. 1976; Pedersen \& Thomsen 1977) today is explained to be the rotation velocity of the star and light variations are considered as due to star spots (see the most recent analysis of Oksala et al. 2015). In fact, it is intriguing that the Catalina Sky Survey found the uhe DO HS 0727+6003 (discussed in Sect. 5.4) to be variable in light with a period of 0.284 days and a photometric amplitude of 0.14 mag (Drake et al. 2014). This short period could well be the rotation period implying that the star may be spotted as well. In fact, Reindl et al. (in prep.) reported both spectral and photometric variability of uhe DOs.

Acknowledgements. We thank R. Napiwotzki for putting the SPY survey spectra at our disposal. T. Rauch was supported by the German Aerospace Center (DLR) under grant 50OR 1507. The TMAD service (http://astro. uni-tuebingen.de/ TMAD) used to compile atomic data for this paper and the TIRO service (http://astro.uni-tuebingen.de/ TIRO), used to generate the model atoms for the iron-group elements, were constructed as part of the activities of the German Astrophysical Virtual Observatory. This research has made use of the SIMBAD database, operated at CDS, Strasbourg, France, 
K. Werner et al.: Metal abundances in hot white dwarfs with signatures of a superionized wind

and of NASA's Astrophysics Data System Bibliographic Services. Some of the data presented in this paper were obtained from the Mikulski Archive for Space Telescopes (MAST). This work had been carried out using the profile fitting procedure OWENS developed by M. Lemoine and the FUSE French Team.

\section{References}

Allard, F., Hauschildt, P. H., Alexander, D. R., Tamanai, A., \& Schweitzer, A 2001, ApJ, 556, 357

Aller, L. H., Appenzeller, I., Baschek, B., et al. 1982, Landolt-Börnstein Numerical Data and Functional Relationships in Science and Technology New Series Gruppe/Group 6 Astronomy and Astrophysics, Vol. 2: Schaifers/Voigt: Astronomy and Astrophysics/Astronomie und Astrophysik Stars and Star Clusters/Sterne und Sternhaufen

Althaus, L. G., Panei, J. A., Miller Bertolami, M. M., et al. 2009, ApJ, 704, 1605

Anderson, L. S. 1989, ApJ, 339, 558

Asplund, M., Grevesse, N., Sauval, A. J., \& Scott, P. 2009, ARA\&A, 47, 481

Barstow, M. A., Barstow, J. K., Casewell, S. L., Holberg, J. B., \& Hubeny, I 2014, MNRAS, 440, 1607

Bashkin, S., \& Stoner, J. O. 1975, Atomic energy levels and Grotrian Diagrams Vol. 1: Hydrogen I - Phosphorus XV, Vol. 2: Sulfur I - Titanium XXII

Cartledge, S. I. B., Clayton, G. C., Gordon, K. D., et al. 2005, ApJ, 630, 355

Dere, K. P., Landi, E., Mason, H. E., Monsignori Fossi, B. C., \& Young, P. R. 1997, A\&AS, 125, 149

Drake, A. J., Graham, M. J., Djorgovski, S. G., et al. 2014, ApJS, 213, 9

Dreizler, S., Heber, U., Napiwotzki, R., \& Hagen, H. J. 1995, A\&A, 303, L53

Gianninas, A., Bergeron, P., Dupuis, J., \& Ruiz, M. T. 2010, ApJ, 720, 581

Good, S. A., Barstow, M. A., Burleigh, M. R., et al. 2005, MNRAS, 363, 183

Groote, D., \& Hunger, K. 1976, A\&A, 52, 303

Groote, D., \& Hunger, K. 1977, A\&A, 56, 129

Groote, D., \& Hunger, K. 1997, A\&A, 319, 250

Hamann, W.-R., Schönberner, D., \& Heber, U. 1982, A\&A, 116, 273

Havnes, O., \& Goertz, C. K. 1984, A\&A, 138, 421

Hébrard, G., \& Moos, H. W. 2003, ApJ, 599, 297

Hébrard, G., Friedman, S. D., Kruk, J. W., et al. 2002, Planet. Space Sci., 50, 1169

Hesser, J. E., Walborn, N. R., \& Ugarte, P. P. 1976, Nature, 262, 116

Hoyer, D., Rauch, T., Werner, K., Kruk, J. W., \& Quinet, P. 2017, A\&A, 598, A 135

Hügelmeyer, S. D., Dreizler, S., Homeier, D., et al. 2006, A\&A, 454, 617

Jeffery, C. S., \& Hamann, W.-R. 2010, MNRAS, 404, 1698

Krtička, J., \& Kubát, J. 2001, A\&A, 377, 175

Krtička, J., \& Kubát, J. 2005, in 14th European Workshop on White Dwarfs, eds

D. Koester, \& S. Moehler, ASP Conf. Ser., 334, 337

Krtička, J., Owocki, S. P., Kubát, J., Galloway, R. K., \& Brown, J. C. 2003, A\&A, 402, 713

Krtička, J., Votruba, V., \& Kubát, J. 2010, A\&A, 516, A100

Krtička, J., Kubát, J., \& Krtičková, I. 2016, A\&A, 593, A101

Kurucz, R. L. 1991, in NATO ASIC Proc. 341: Stellar Atmospheres - Beyond Classical Models, eds. L. Crivellari, I. Hubeny, \& D. G. Hummer, 441
Kurucz, R. L. 2009, in AIP Conf. Ser. 1171, eds. I. Hubeny, J. M. Stone, K. MacGregor, \& K. Werner, 43

Kurucz, R. L. 2011, Can. J. Phys., 89, 417

Landi, E., Young, P. R., Dere, K. P., Del Zanna, G., \& Mason, H. E. 2013, ApJ, 763,86

Lemoine, M., Vidal-Madjar, A., Hébrard, G., et al. 2002, ApJS, 140, 67

Napiwotzki, R. 1997, in The Third Conference on Faint Blue Stars, eds. A. G. D. Philip, J. Liebert, R. Saffer, \& D. S. Hayes, 207

Napiwotzki, R., \& Rauch, T. 1994, A\&A, 285, 603

Napiwotzki, R., Christlieb, N., Drechsel, H., et al. 2003, The Messenger, 112, 25

Oksala, M. E., Kochukhov, O., Krtička, J., et al. 2015, MNRAS, 451, 2015

Pedersen, H., \& Thomsen, B. 1977, A\&AS, 30, 11

Rauch, T., \& Deetjen, J. L. 2003, in Stellar Atmosphere Modeling, eds. I. Hubeny, D. Mihalas, \& K. Werner, ASP Conf. Ser., 288, 103

Rauch, T., Gamrath, S., Quinet, P., et al. 2017, A\&A, 599, A142

Reindl, N., Rauch, T., Werner, K., et al. 2014a, A\&A, 572, A117

Reindl, N., Rauch, T., Werner, K., Kruk, J. W., \& Todt, H. 2014b, A\&A, 566, A116

Seaton, M. J., Yan, Y., Mihalas, D., \& Pradhan, A. K. 1994, MNRAS, 266, 805

Smith, M. A., \& Bohlender, D. A. 2007, A\&A, 475, 1027

Sofia, U. J., Wolff, M. J., Rachford, B., et al. 2005, ApJ, 625, 167

Springmann, U. W. E., \& Pauldrach, A. W. A. 1992, A\&A, 262, 515

Unglaub, K. 2006, Baltic Astron., 15, 147

Unglaub, K. 2008, A\&A, 486, 923

Unglaub, K., \& Bues, I. 2000, A\&A, 359, 1042

Voigt, H.-H. 2012, Abriss der Astronomie, 6, Auflage (Wiley-VCH)

Werner, K. 1996, ApJ, 457, L39

Werner, K., \& Dreizler, S. 1999, J. Comput. Appl. Math., 109, 65

Werner, K., Dreizler, S., Heber, U., et al. 1995, A\&A, 293, L75

Werner, K., Dreizler, S., Heber, U., \& Rauch, T. 1997a, in White dwarfs, eds. J. Isern, M. Hernanz, \& E. Garcia-Berro, Astrophys. Space Sci. Lib., 214, 207

Werner, K., Dreizler, S., Rauch, T., \& Heber, U. 1997b, in The Third Conference on Faint Blue Stars, eds. A. G. D. Philip, J. Liebert, R. Saffer, \& D. S. Hayes, 227

Werner, K., Dreizler, S., Rauch, T., et al. 1999, in 11th European Workshop on White Dwarfs, eds. S.-E. Solheim, \& E. G. Meistas, ASP Conf. Ser., 169, 511

Werner, K., Deetjen, J. L., Dreizler, S., et al. 2003a, in Stellar Atmosphere Modeling, eds. I. Hubeny, D. Mihalas, \& K. Werner, ASP Conf. Ser., 288, 31

Werner, K., Dreizler, S., Kruk, J. W., \& Sitko, M. L. 2003b, in NATO ASIB Proc. 105: White Dwarfs, eds. D. de Martino, R. Silvotti, J.-E. Solheim, \& R. Kalytis, 105, 171

Werner, K., Dreizler, S., \& Rauch, T. 2012, Astrophysics Source Code Library, [record ascl: 1212.015]

Werner, K., Rauch, T., \& Kepler, S. O. 2014, A\&A, 564, A53

Werner, K., Rauch, T., \& Kruk, J. W. 2015, A\&A, 582, A94

Werner, K., Rauch, T., \& Kruk, J. W. 2017, A\&A, 601, A8

Ziegler, M., Rauch, T., Werner, K., Köppen, J., \& Kruk, J. W. 2012, A\&A, 548, A109 